\title{
Observational Prospects for Afterglows of Short- Duration Gamma-Ray Bursts
}

\section{Citation}

Panaitescu, A., P. Kumar, and R. Narayan. 2001. “Observational Prospects for Afterglows of Short-Duration Gamma-Ray Bursts." The Astrophysical Journal 561 (2): L171-74. https:// doi.org/10.1086/324678.

\section{Permanent link}

http://nrs.harvard.edu/urn-3:HUL.InstRepos:41384937

\section{Terms of Use}

This article was downloaded from Harvard University's DASH repository, and is made available under the terms and conditions applicable to Other Posted Material, as set forth at http:// nrs.harvard.edu/urn-3:HUL.InstRepos:dash.current.terms-of-use\#LAA

\section{Share Your Story}

The Harvard community has made this article openly available.

Please share how this access benefits you. Submit a story.

Accessibility 


\title{
OBSERVATIONAL PROSPECTS FOR AFTERGLOWS OF SHORT DURATION GAMMA-RAY BURSTS
}

\author{
A. Panaitescu \\ Dept. of Astrophysical Sciences, Princeton University, Princeton, NJ 08544 \\ P. KUMAR \\ Institute for Advanced Study, Olden Lane, Princeton, NJ 08540 \\ R. NARAYAN \\ Harvard-Smithsonian Center for Astrophysics, Cambridge, MA 02138 \\ Draft version October 31, 2018
}

\begin{abstract}
If the efficiency for producing $\gamma$-rays is the same in short duration ( $\lesssim 2 \mathrm{~s})$ Gamma-Ray Bursts (GRBs) as in long duration GRBs, then the average kinetic energy of short GRBs must be $\sim 20$ times less than that of long GRBs. Assuming further that the relativistic shocks in short and long duration GRBs have similar parameters, we show that the afterglows of short GRBs will be on average 10-40 times dimmer than those of long GRBs. We find that the afterglow of a typical short GRB will be below the detection limit ( $\lesssim 10 \mu \mathrm{Jy})$ of searches at radio frequencies. The afterglow would be difficult to observe also in the optical, where we predict $R \gtrsim 23$ a few hours after the burst. The radio and optical afterglow would be even fainter if short GRBs occur in a low-density medium, as expected in NS-NS and NS-BH merger models. The best prospects for detecting short-GRB afterglows are with early ( $\lesssim 1$ day) observations in X-rays.

Subject headings: gamma-rays: bursts - ISM: jets and outflows - radiation mechanisms: non-thermal
\end{abstract}

\section{INTRODUCTION}

Kouveliotou et al. (1993) showed that the durations of Gamma Ray Bursts (GRBs) have a bimodal distribution, with roughly a third of the observed bursts corresponding to a short duration $(\lesssim 2 \mathrm{~s})$ population, and the remaining two-thirds belonging to a long duration ( $22 \mathrm{~s}$ ) population.

Searches for transient $\mathrm{X}$-ray, optical and radio afterglow emission have so far been largely limited to long duration bursts. This is because the Italian-Dutch BeppoSAX satellite and other presently active instruments, which have been used to obtain well-determined GRB coordinates, are sensitive only to bursts longer than a few seconds. Most of the X-ray follow-ups of long GRBs have been successful, and 35 X-ray afterglows have been detected so far (mid-2001). The success rate of optical searches has been somewhat less (Lazzati, Covino \& Ghisellini 2001; Fynbo et al. 2001), and 23 optical afterglows have been detected; the majority of optical afterglows have yielded redshifts. The success rate in radio has been comparable to that in the optical.

Among short GRBs, optical and radio searches for afterglows have so far been carried out for only four sources that happened to be well localized by the Interplanetary Network (Hurley et al. 2001). No afterglow emission was detected, but the sensitivity of the searches was not very high. More searches will be carried out in the future when rapid arc-minute localizations of short GRBs become routinely available from the upcoming HETE II and Swift satellites.

In this Letter, we make reasonable assumptions about the physical parameters of short GRBs and estimate the broadband afterglow emission to be expected from these bursts. The results may be useful for designing search strategies for afterglows of short GRBs.

\section{SHORT VERSUS LONG GRBS}

The predictions for the light-curves of the afterglows depend on some key parameters. We attempt to estimate the relative magnitudes of these parameters in short and long GRBs using observational data and some theoretical ideas.

Studies of the temporal and spectral properties of short and long GRBs have revealed that the burst duration is anticorrelated with the spectral hardness (Kouveliotou et al. 1993). The peak frequency of the $\nu F_{\nu}$ spectrum increases with the bulk Lorentz factor of the GRB in the external shock model for producing $\gamma$-ray emission (Rees \& Mészáros 1992; Piran, Shemi \& Narayan 1993; Katz 1994), however the relationship is complicated in the internal shock model (see Piran 1999 for a review). Fortunately, the Lorentz factor (LF) of the late-time GRB remnant which produces the afterglow is practically independent of the LF during the early GRB phase, and so it is unlikely that any difference in the initial LF for the long and the short duration GRBs will have any effect on the afterglow flux. We note, however, that if the initial jet opening angle $\theta_{j}$ for short duration GRBs were larger than longer lasting GRBs, it will cause the afterglows of short GRBs to become considerably dimmer since the jet transition time ( $\operatorname{see} \S 3$ ) varies as $t_{j} \propto \theta_{j}^{8 / 3}$.

By analysing a sample of over $400 \mathrm{GRBs}$, and allowing for observational bias against detecting weak and long bursts with peak fluxes below the detection threshold, Lee \& Petrosian (1997) showed that there is a highly significant positive correlation between the burst fluence and duration. In another study, Mukherjee et al. (1998) used two multivariate clustering methods to show that most of the structure in the multidimensional space of burst observations is contained in three fundamental quantities: duration, fluence, and spectral hardness. According to their analysis, short/hard GRBs have a $25 \mathrm{keV}-1 \mathrm{MeV}$ fluence $\Phi$ (in cgs units) of approximately $\log \Phi=-6.4 \pm 0.6$, while long/soft GRBs have $\log \Phi=-5.2 \pm 0.6$. Short bursts thus have about 20 times less fluence than long bursts (see also Mao et al. 1994, Piran 1996). 
Figure 1 shows the $25 \mathrm{keV}-1 \mathrm{MeV}$ fluences $\Phi$ and the durations $T_{90}$ of 34 long GRBs for which afterglows have been observed. The average duration for these GRBs is $\log T_{90}=$ $1.5 \pm 0.5$, similar to that of the 486 long bursts analyzed by Mukherjee et al. (1998) from the Third BATSE Catalog (Meegan et al. 1996). The average fluence of long GRBs with afterglows is $\log \Phi=-4.8 \pm 0.72$, implying that these bursts are, on average, approximately twice as bright as the long bursts analyzed by Mukherjee et al. (1998) and 40 times more energetic than the 203 short bursts analyzed by them.

The GRB energy output is determined by the kinetic energy of the relativistic outflow and the efficiency with which dissipative and radiative mechanisms convert some of the energy into $\gamma$-ray emission. Assuming that GRBs arise from internal shocks in unsteady winds, the dissipation efficiency is determined by the magnitude of the fluctuations in the ejection Lorentz factors of various parts of the outflow. Our present understanding of the properties of GRB progenitors does not allow us to establish a correlation between the GRB duration and efficiency. It is possible that short GRBs are somewhat less efficient than long GRBs because the radius at which internal shocks occur in short GRBs is closer to the photospheric radius (Kumar 1999), so that some of the emission may be degraded by multiple scattering. Ignoring this effect, we expect the ratio of the kinetic energies of short and long GRBs to be the ratio of their fluences, i.e., a factor of about 20.

We finally discuss the environments in which short and long bursts take place. The existence of these two clearly distinct populations of GRBs probably implies different physical origins. Most models of GRBs - mergers of binary neutron stars (NS-NS), black hole (BH)-NS, or BH-white dwarf (WD), and failed supernovae/collapsars - involve the formation of a $\mathrm{BH}$ surrounded by a disk of debris. The spin energy of the hole and the gravitational, thermal, and rotational energy of the disk represent the available reservoirs to power the $\gtrsim 10^{51} \mathrm{ergs}$ required for the GRB . The BH spin may be tapped by the BlandfordZnajek process (1977), and the disk energy may be extracted either via neutrino annihilation (Eichler et al. 1989) or via magnetic fields/flares (Narayan, Paczyński \& Piran 1992; see e.g. Mészáros, Rees \& Wijers 1999 for a review).

The burst duration is determined both by the timescale for ejecting the relativistic outflow, and by the processes that $i$ ) shape the outflow dynamics until the gas reaches the region where the $\gamma$-ray photons are emitted, such as the penetration of the GRB jet through the envelope of a collapsed star, and $i i$ ) convert the outflow kinetic energy into $\gamma$-rays. In those bursts where the high energy emission arises as the outflow energy is dissipated through interaction with the circumburst medium, GRBs of long duration can be obtained even if the initial ejection is impulsive. However, the general absence of signatures of an external shock in the temporal structure of GRBs (Sari \& Piran 1997; Ramirez-Ruiz \& Fenimore 2000) indicates that the GRB emission is produced in internal shocks over a small range of distances. If this is the case, then the GRB duration is a direct measure of the time interval during which the "central engine" is active.

If GRBs originate in NS-NS or NS-BH mergers (Goodman 1986; Eichler et al. 1989; Paczyński 1991; Narayan et al. 1992; Mészáros \& Rees 1992; Katz \& Canel 1996), enough mass reaches the resulting $\mathrm{BH}$ to power the GRB, provided the disk is sufficiently small and accretion is driven by neutrino cooling (Narayan, Piran \& Kumar 2001). The expected duration of the relativistic wind (and thus the GRB) is under $1 \mathrm{~s}$. Thus these progenitors can naturally produce short GRBs.

The collapse of the iron core of a massive star (collapsar model: Woosley 1993; Paczyński 1998) with intermediate angular momentum leads to the formation of a disk with a sufficient accretion rate to power a relativistic outflow lasting for 10-20 s (MacFadyen \& Woosley 1999). The collapsar model is thus more appropriate for long GRBs. If the core collapse produces a NS and an out-going shock, the $\mathrm{BH}$ and the torus form when the ejected matter, lacking sufficient momentum, falls back (MacFadyen, Woosley \& Heger 2001). In this model the GRB duration is set by the dynamics of the fallback, and most likely would accommodate only the longest GRBs, lasting for more than $100 \mathrm{~s}$. Long GRBs could also arise from WD-BH (Fryer et al. 1999) or helium star-BH mergers (Zhang \& Fryer 2001), which lead to the formation of larger disks with accretion times above $10 \mathrm{~s}$. However, it is likely that the gas will accrete via a convection-dominated accretion flow in these systems, in which case the flow is likely to be rather inefficient at extracting the disk energy (Narayan et al. 2001).

According to these ideas, then, there is a clear association between the type of GRB progenitor and the burst duration. Short GRBs are likely to involve merging NS binaries (NSNS or NS-BH), which should occur predominantly in the low density halo of the host galaxy, given the velocities of a few hundred $\mathrm{km} \mathrm{s}^{-1}$ acquired by the NS at birth and the binary coalescence time of about 100 Myr. Long GRBs, on the other hand, are likely to be associated with massive stars that at the end of their evolution (a few Myr) are still within the cloud where they formed. We note that Bloom, Kulkarni \& Djorgovski (2001) have identified the host galaxies of 20 afterglows of long duration GRBs and have found GRB-host offsets lower than those expected for merging NS binaries (Bloom, Sigurdsson \& Pols 1999; Bulik, Belczyński \& Zbijewski 1999; Fryer, Woosley \& Hartmann 1999). Furthermore, the high column densities identified by Owens et al. (1998) and Galama \& Wijers (2001) from the absorption of the X-ray afterglow emission indicate that long-duration GRBs occur in giant molecular clouds, yielding further support for the massive star collapse model for long bursts.

If the above arguments are correct, we expect the afterglows of short GRBs to occur in a more tenuous medium compared to the afterglows of long-duration GRBs. The difference in the density of the medium is likely to be a few orders of magnitude.

\section{AFTERGLOWS OF SHORT GRBS}

Given the kinetic energy $E$ of the relativistic outflow and the number density $n$ of the ambient external medium, plus a few other dimensionless parameters described below, the afterglow light-curves in various bands may be theoretically estimated (e.g., Sari, Piran \& Narayan 1998; Panaitescu \& Kumar 2001a, and references therein). If we consider early times, when the radio emission is below the peak frequency $\nu_{p}$ of the afterglow spectrum, and if we assume that the cooling frequency $\nu_{c}$ is between the optical and X-ray bands, then the analysis of Panaitescu \& Kumar (2001a) shows that

$$
\begin{gathered}
F_{\text {radio }} \propto \varepsilon_{e}^{-2 / 3} \varepsilon_{B}^{1 / 3} E^{5 / 6} n^{1 / 2}, \\
F_{\text {optical }} \propto \varepsilon_{e}^{p-1} \varepsilon_{B}^{(p+1) / 4} E^{(p+3) / 4} n^{1 / 2}, \\
F_{X-\text { ray }} \propto \varepsilon_{e}^{p-1} \varepsilon_{B}^{(p-2) / 4} E^{(p+2) / 4} .
\end{gathered}
$$

Here, $E$ is the isotropic equivalent kinetic energy of the outflow, $\varepsilon_{e}$ is the fractional energy in electrons, $p$ is the index 
of the power-law distribution of the electron Lorentz factor $N(\gamma) \propto \gamma^{-p}$, and $\varepsilon_{B}$ is the fraction of the post-shock thermal energy in magnetic field (see Panaitescu \& Kumar 2001a for details). The break frequencies satisfy $\nu_{p} \propto E^{1 / 2} \varepsilon_{e}^{2} \varepsilon_{B}^{1 / 2}$, $\nu_{c} \propto E^{-1 / 2} n^{-1} \varepsilon_{B}^{-3 / 2}$ for synchrotron-dominated electron cooling, and $\nu_{c} \propto\left[E^{-p / 2} n^{-2} \varepsilon_{e}^{-2(p-1)} \varepsilon_{B}^{-p / 2}\right]^{1 /(4-p)}$ when inverse Compton losses exceed synchrotron cooling.

The above equations show that for $p \sim 2$, which is the average value for this parameter determined by Panaitescu \& Kumar (2001b) from numerical modeling of eight GRB afterglows, the afterglow flux is roughly proportional to the kinetic energy $E$. Since short GRBs have a fluence on average $\sim 20$ times smaller than that of long GRBs, other parameters being equal, we expect the afterglows of short GRBs to be $\sim 20$ times dimmer than the afterglows of long GRBs.

The equations show that the afterglow flux in short bursts would be further suppressed if these bursts occur in a lower density medium compared to long bursts. The expressions for the radio and optical flux have an explicit dependence on $n$. Even though the formula for the X-ray flux does not have an explicit dependence, this flux is also suppressed since, for low $n$, the cooling frequency $\nu_{c}$ moves above the $\mathrm{X}$-ray band and so the $\mathrm{X}$-ray afterglow light-curve is described by equation (2) rather than equation (3).

Finally, if the breaks observed at one to a few days in the optical emission of several GRB afterglows are due to collimation of the outflow (Rhoads 1999; Sari et al. 1999; Kumar \& Panaitescu 2000), then the $\sim 20$ times smaller kinetic energy of the jets in short GRBs will cause the jet break time $t_{j} \propto E^{1 / 3}$ to occur $\sim 3$ times earlier in these bursts compared to long GRBs. This will further diminish the brightness of short-GRB afterglows (at a fixed observing time) by a factor of about $2-3$.

These conclusions are illustrated in Figure 2, which compares the radio, optical, and X-ray emission of a typical longGRB afterglow with that predicted for short-GRB afterglows with lower values of $E$ and $n$. The numerical calculations of the afterglow dynamics and emission of radiation were done by the methods described in Panaitescu \& Kumar (2000). The parameters for the long-GRB afterglow were chosen such that at one day after the burst the model yields a radio flux of $\sim 0.5$ mJy, an optical brightness of $R \sim 20$ and a $2-10 \mathrm{keV}$ X-ray flux of $\sim 10^{-11} \mathrm{erg} \mathrm{cm}^{-2} \mathrm{~s}^{-1}$, in rough agreement with observations.

We see from Fig. 2 that the radio emission from a typical short-GRB afterglow will most likely be very difficult to detect. The same is true also for the optical emission, where only early ( $\lesssim 1 / 2$ day) observations as deep as the measurements made by Groot et al. (1998) for GRB 970828 and by Fynbo et al. (2001) for GRB 000630 are likely to be successful. The best chance for detecting the afterglow of a short GRB is with $\mathrm{X}$-ray observations. BeppoSax will need to observe earlier than $\sim 1 / 2$ day, and CXO, HETE II and Swift earlier than $\sim 2$ days. Such observations are quite feasible.

Because of the roughly linear dependence of the afterglow brightness on the kinetic energy $E$, any difference in the aver- age redshifts of short and long GRBs will not alter the above conclusions. The redshift enters into the estimate of $E$, and through it in the estimate of the afterglow luminosity, but it factors out when the luminosity is converted to the observed flux.

\section{CONCLUSIONS}

The fluences above $25 \mathrm{keV}$ of GRBs detected by BATSE are positively correlated with the durations of these bursts; long bursts (durations longer than a few seconds) are on average 20 times more energetic than short bursts. Moreover, the $\sim$ three dozen long GRBs for which afterglows have been detected so far (following their accurate localization by BeppoSAX and the Interplanetary Network) are on average 40 times brighter (in fluence) than typical short GRBs. Thus we expect the relativistic outflow of a long GRB to have about 20 times the kinetic energy of the outflow from a short GRB. If the other parameters that influence the dynamics of the GRB remnant and the emission of radiation are roughly the same, we predict that the afterglows of short GRBs should be a factor of 10 or more dimmer than the afterglows of long GRBs.

If short GRBs arise from merging NS-NS or NS-BH binaries which may have traveled upto or beyond the very tenuous outskirts of their host galaxies, and if long GRBs are due to the core collapse of massive stars which die in the dense molecular clouds where they were formed, then the densities $n$ of the media surrounding these two types of GRBs may differ by a few orders of magnitude. This could further seriously diminish the prospects of detecting radio and optical afterglows of short GRBs since the afterglow brightness in these bands is proportional to $n^{1 / 2}$.

We conclude that the best chance of detecting afterglows of short GRBs is with early X-ray observations within 1 day after the GRB. Rapid and deep optical follow-up within a few hours after the main event may also lead to a detection. Radio observations appear the least promising (Fig. 2).

Of course, short GRBs significantly brighter than average could be as energetic as some of the long GRBs for which afterglows have been seen (Fig. 1). The afterglows of such unusually bright short GRBs could be detected even beyond a day, particularly if the magnetic field strength is close to equipartition $\left(\varepsilon_{B} \rightarrow 1\right)$. But such bursts should be in the minority. On the other hand, if future observations indicate that the afterglows of most short GRBs are as bright as those of long GRBs, it would imply that one or more of the assumptions we have made in our analysis is invalid. One possibility is that the $\gamma$-efficiency is significantly lower in short bursts than in long bursts, as disussed in Kumar (1999), and another is that the efficiency spans a wide range in both types of bursts, indicating a highly inhomogeneous outflow (Kumar \& Piran 2000).

AP acknowledges support from Princeton University through the Lyman Spitzer, Jr. fellowship. PK was supported in part by the Ambros Monell Foundation and NSF grant PHY-0070928, and RN was supported in part by NSF grant AST-9820686. We are grateful to Tsvi Piran for comments.

\section{REFERENCES}

Eichler, D. et al. 1989, Nature, 340, 126

Fryer, C., Woosley, S., Herant, M \& Davies, M. 1999, ApJ, 520, 650

Fynbo, J. et al. 2001, A\&A 369, 373

Goodman, J. 1986, ApJ, 308, L17

Groot, P. et al. 1998, ApJ, 493, L27
Hurley, K. et al. 2001, ApJ, submitted astro-ph/0107188

Katz, J. 1994, ApJ, 422, 248

Katz, J. \& Canel, L. 1996, ApJ, 471, 915

Kouveliotou, C. et al. 1993, ApJ, 413, L101

Kumar, P. 1999, ApJ, 523, L113 
Kumar, P. \& Piran, T. 2000, ApJ, 535, 152

Kumar, P. \& Panaitescu, A. 2000, ApJ, 541, L9

Lazzati, D., Govino, S \& Ghisellini, \& 2001, A\& s, submitted astro$\mathrm{ph} / 0011443)$

Lee, T. \& Petrosian, V. 1997, ApJ, 474, 37

MacFadyen, A. \& Woosley, S. 1999, ApJ, 524, 262

MacFadyen, A., Woosley, S. \& Heger, A. 2001, ApJ, 550, 410

Mao, S., Narayan, R., and Piran, T., 1994, ApJ, 420, 171

Meegan, C. et al. 1996, ApJ, S106

Mészáros, P. \& Rees, M. 1992, ApJ, 397, 570

Mészáros, P., Rees, M.J. \& Wijers, R. 1999, New Ast, 4, 303

Mukherjee, S. et al. 1998, ApJ, 508, 314

Narayan, R., Paczyński, B. \& Piran, T. 1992, ApJ, 395, L83

Narayan, R., Piran, T. \& Kumar, P. 2001, ApJ, in press astro-ph/0103360)

Paczyński, B. 1991, Acta Ast, 41, 257

Paczyński, B. 1998, ApJ, 494, L45

Panaitescu, A. \& Kumar, P. 2000, ApJ, 543, 66
Panaitescu, A. \& Kumar, P. 2001a, ApJ, 554, 667

Panaitescu, A. \& Kumar, P. 2001b, ApJ, submitted

Piran, T. 1996, in "Compact stars in binaries", proceedings from IAU symposium 165, Eds J. van Paradijs, E. P. J. van den Heuvel, E. Kuulkers. Kluwer Academic Publishers, Dordrecht, p.489

Piran, T. 1999, Phys. Rep., 314, 575

Piran, T., Shemi, A. \& Narayan, R. 1993, MNRAS, 263, 861

Ramirez-Ruiz, E. \& Fenimore, E. 2000, ApJ, 539, 712

Rees, M.J. \& Mészáros, P. 1992, MNRAS, 258, 41p

Rhoads, J. 1999, ApJ, 525, 737

Sari, R., Piran, T. 1997, ApJ, 485, 270

Sari, R., Piran, T., \& Halpern, J. 1999, ApJ, 519, L17

Sari, R., Piran, T., \& Narayan, R. 1998, ApJ, 497, L17

Tavani, M. 1998, ApJ, 497, L21

Woosley, S. 1993, ApJ, 405, 273

Zhang, W. \& Fryer, C. 2001, ApJ, 550, 357

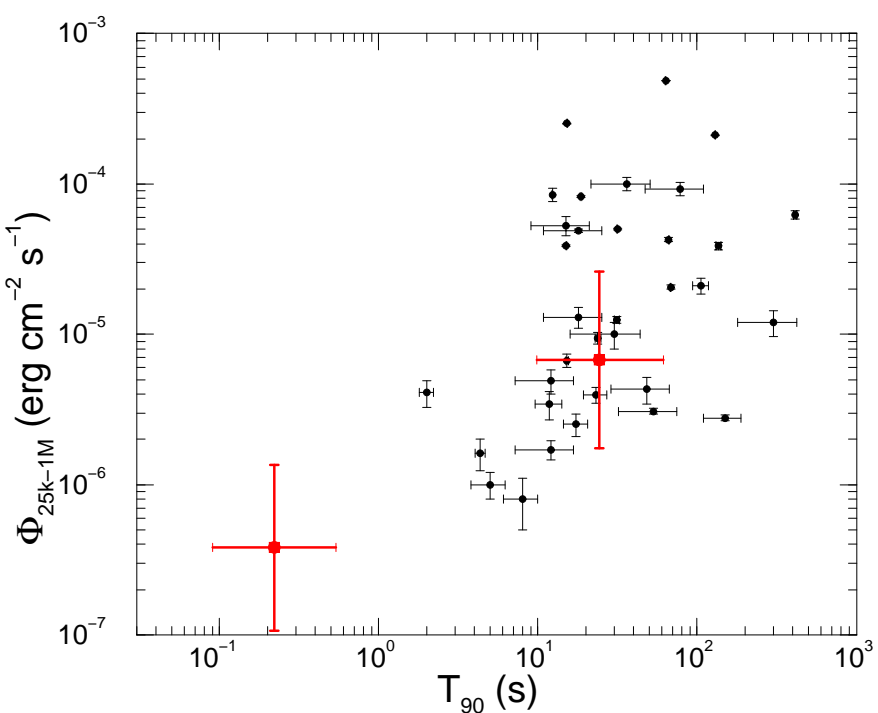

FIG. 1. - $25 \mathrm{keV-1} \mathrm{MeV} \mathrm{fluence} \Phi$ versus duration $T$ for 34 long GRBs for which a transient emission (afterglow) has heen ohserved Data for 21 GRBs

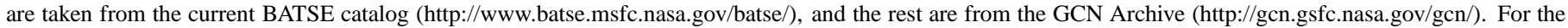

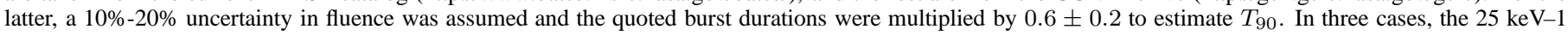
$\mathrm{MeV}$ fluence was calculated from the reported $25-100 \mathrm{keV}$ fluence assuming a spectral slope of -1 . Squares with large error bars show the average fluences and

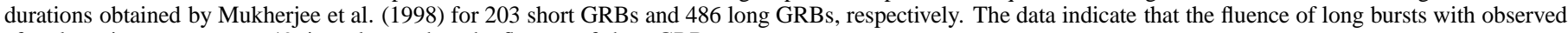
afterglows is on average $\sim 40$ times larger than the fluence of short GRBs. 

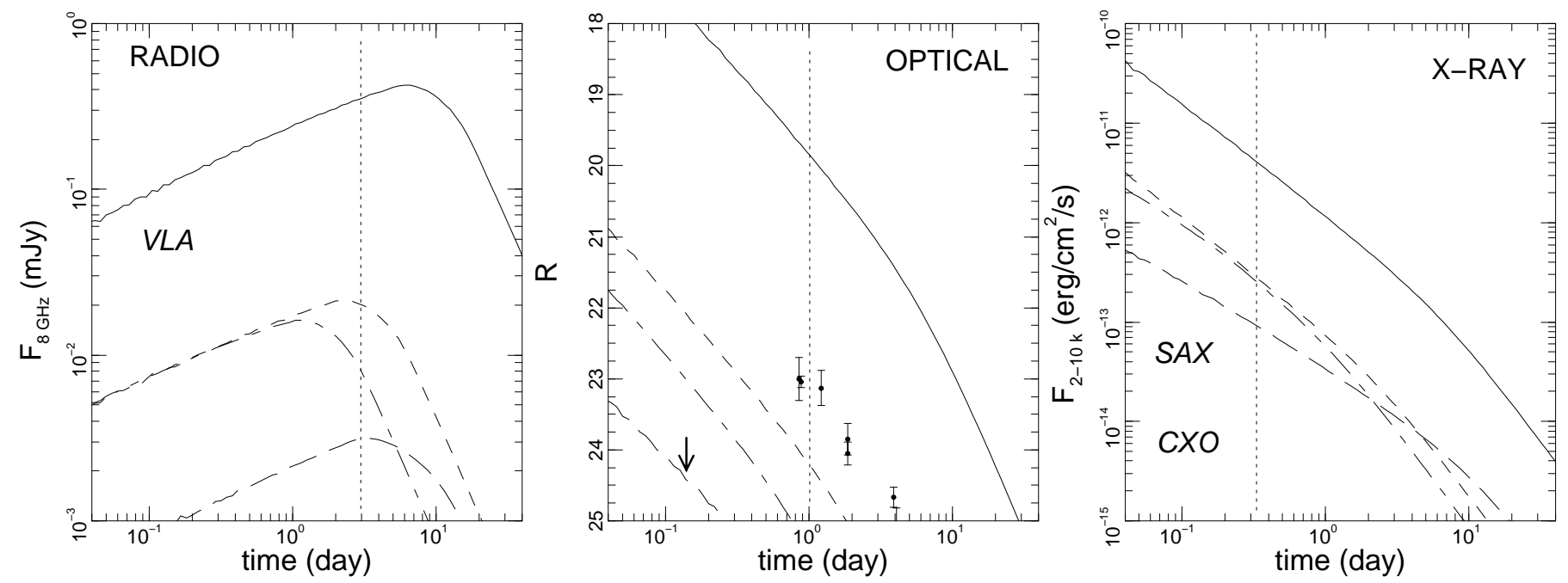

FIG. 2.- The solid lines show the radio ( $8 \mathrm{GHz})$, optical (R-band) and X-ray fluxes (2-10 keV band) corresponding to a model afterglow of a long GRB. Model parameters are (see text): $n=0.1 \mathrm{~cm}^{-3}, \varepsilon_{e}=0.03, \varepsilon_{B}=10^{-3}, p=2.0$, redshift $z=1$, isotropic equivalent kinetic energy $E=10^{53}$ erg. The relativistic ejecta were assumed to be collimated, with an initial aperture (half-angle) of $0.1 \mathrm{rad}$. The collimation causes the breaks seen in some of the light-curves. The short-dashed lines show the model light-curves of a short GRB, for which all the parameters are the same except that $E=5 \times 10^{51}$ erg. The long-dashed lines correspond to another short-GRB model in which $n=10^{-3} \mathrm{~cm}^{-3}$, more characteristic of the low density environment in which these bursts may occur if they are associated with binary mergers. The dot-dashed lines are for a short GRB with a lower redshift $(z=0.5)$ and correspondingly less energy ( $E=10^{51}$ erg), as suggested by the higher value of $\left\langle V / V_{\max }\right\rangle$ for short GRBs estimated by Katz \& Canel (1996), Piran (1996) and Tavani (1998). Also indicated on the plots are the sensitivities of the best radio and X-ray instruments currently available, the optical upper limit (arrow) obtained by Groot et al. (1997) for the afterglow of GRB 970828 ( $R>23.8$ at 4 hours), and the light-curve of the very dim afterglow of the long GRB 000630 (Fynbo et al. 2001). The vertical dotted lines indicate the time after the initial GRB when afterglow observations are typically made. 